\title{
Highly Permeable Membranes for Live Cell Imaging of Co-Cultures
}

\author{
Thomas R. Gaborski*, Barrett J. Nehilla**, Christopher C. Striemer* and James L. McGrath** \\ * SiMPore, Inc., 150 Lucius Gordon Drive, Suite 100, West Henrietta, NY 14586 \\ ** Department of Biomedical Engineering, University of Rochester, Rochester, NY 14627
}

A new class of porous membrane has been fabricated that is unique in its combination of nanoscale thickness $(5-30 \mathrm{~nm})$ with macroscopic, yet robust, millimeter-scale lateral dimensions and tunable pore sizes in the range of 5 to $100 \mathrm{~nm}$. The membrane material is porous nanocrystalline Si (pnc-Si), first reported in a 2007 Nature paper by researchers at the University of Rochester [1] and now being scaled-up to commercial volumes by a startup company, SiMPore, Inc. Traditional separation membranes with pores in this size regime are typically polymeric materials that are microns in thickness, leading to pore morphologies that resemble long narrow tubes or tortuous-path 3-D matrices. In an ideal membrane, thickness approaches the length-scale of pore diameters resulting in a simplified structure of holes in a thin sheet. Such porous films are predicted by classical transport theories to have rapid diffusive and forced flow properties. Indeed measurements of pnc-Si permeability match theory and are significantly greater than other nanoporous materials [2].

In addition to use in separations and purification, pnc-Si has unique applications in cell biology. The silicon membrane naturally oxidizes under ambient conditions creating a glass-like surface that is amenable to growth of numerous cell types. Pnc-Si offers a physical barrier for cell co-culture and drug permeability applications while minimizing the resistance to diffusion of small molecules (Figure 1). Unlike conventional polymeric membranes that are microns in thickness, the thinness of the membrane results in nearly optical transparency, enabling high-resolution imaging of cells on both the top and the bottom of the membrane (Figure 2). In addition, membrane surface treatments can be used to control degradation from 1 to 30 days yielding a two-dimensional degradable scaffold. In combination, these characteristics enable an ideal support structure for engineering of layered tissues.

We have completed preliminary studies comparing cell adhesion and growth rates of human umbilical vein endothelial cells (HUVEC) on pnc-Si, tissue culture plastic and glass surfaces. We find no significant differences between these substrates, while negative controls on Teflon coated surfaces show reduced adhesion and growth. We are currently developing an improved blood-brain barrier co-culture model using brain endothelial and glial cells separated and supported by a pnc-Si membrane. The goals of the study are to demonstrate improved in vitro transendothelial electrical resistance (TEER) and permeability measurements to provide a better model system for drugs targeted to the brain.

\section{References}

[1] C.C. Striemer et. al., Nature. 445 (2007) 749.

[2] E. Kim et. al., J Am Chem Soc. 130 (2008), 4230.

[3] This research was in part supported by the Center for Emerging and Innovative Sciences, New York State Foundation for Science, Technology and Innovation. 

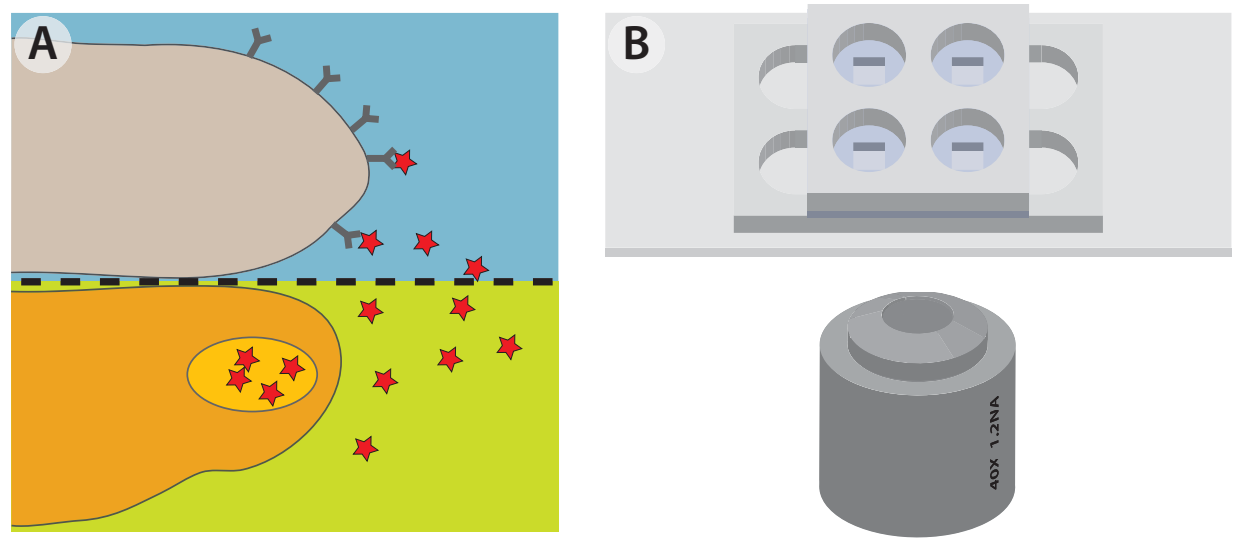

FIG. 1. A) Ultrathin silicon membranes are highly permeable and bring two cell populations within $30 \mathrm{~nm}$, while maintaining physical separation. This enables ideal co-culture conditions for short distance signaling, while simplifying recovery of either cell population without the need for cell sorting. B) Membranes can be patterned in many geometries including miniature arrays for live-cell fluorescent imaging of co-cultures.
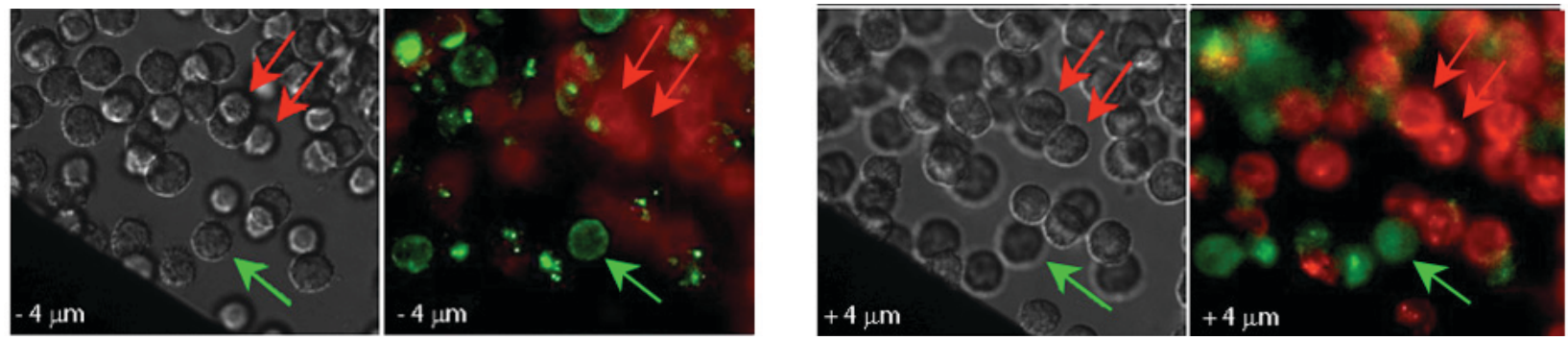

FIG. 2. Ultrathin silicon membranes are optically transparent. One population of neutrophils was labeled with anti-L-selectin (Alexa488-Green) and plated on the bottom of the membrane, while another population was labeled with anti-L-selectin (Alexa546-Red) and plated on the top of the membrane. Z-slices illustrate that the green population is in focus at -4 microns (neutrophil diameter $\sim 8$ microns), while the red population is in focus at +4 microns. The membrane is transparent and is not directly discernible at any focal plane. 